\title{
Skin Picking in Atopic Dermatitis and Psoriasis Patients: A Single-centre Cross-sectional Study in China
}

Yanjiao JU1 ${ }^{1}$, Mufeng $\mathrm{LI}^{2}$, Xueyan $\mathrm{LU}^{1}$, Cheng $\mathrm{ZHAO}^{3}$, Chunlei ZHANG ${ }^{1}$ and Zhiqiang XIE ${ }^{1 *}$

${ }^{1}$ Department of Dermatology, Peking University Third Hospital, No. 49 Huayuan North Road, Haidian District, Beijing 100191, ${ }^{2}$ Department of Dermatology, Peking University Shougang Hospital, Beijing and ${ }^{3}$ Department of Vascular Surgery, Qingdao Municipal Hospital, Qingdao, China. *E-mail: xiezq66@163.com

Accepted Dec 14, 2021; Epub ahead of print Dec 14, 2021

Chronic pruritus $(\mathrm{CP})$ is a predominant feature of many inflammatory dermatoses, such as atopic dermatitis (AD) and psoriasis (1). Scratching is a natural response to itching. Interestingly, scratching in patients with $\mathrm{CP}$ can induce overactivity in motor-related regions and reward system; hence it can be pleasurable, rewarding, and even addictive (2), which leads to the clinically challenging "itch-scratch cycle". Skin picking is generated as a response to pruritus or other skin sensations, such as burning, tingling or pain. Behaviours of skin picking include scratching, biting, rubbing or digging the skin, removing skin parts, and pinching (3). In pathological state, impulsive-compulsive behaviours arise subsequently, and with anxiety and tension, which make skin picking difficult to control (4). This leads to severe self-injurious skin picking, termed skin picking disorder (SPD). Dermatological and/or medical differential diagnoses may include severe pruritus that invariably leads to skin-picking behaviour, such as scabies, but acne frequently associated with a comorbid SPD (5). Skin picking behaviours of patients with CP have not been well studied. The Skin Picking Scale (SPS), compiled by Keuthen et al. in 2001 (6), is a 6-item measure for the assessment of skin picking. A total score of 7 differentiates severe self-injurious from nonself-injurious skin pickers (6). The aim of this study was to investigate the self-injurious skin picking behaviours of AD and psoriasis patients using SPS. A secondary aim was to examine the overall association of degree of skin picking with itch and skin lesion intensity.

\section{MATERIALS, METHODS AND RESULTS}

This study used cross-sectional survey data collected from 122 adult patients with AD and 216 adult patients with psoriasis at the outpatient clinic of Department of Dermatology, Peking University Third Hospital, Beijing from January 2018 to December 2020. Diagnosis AD was made according to wellestablished Hanifin \& Rajka criteria, while diagnosis of psoriasis was based on clinical manifestation (in doubtful cases biopsies were taken). None of the patients had a known diagnosed primary psychological disorder. All the patients completed the Chinese version of Skin Picking Scale (SPS) (6). In 2017, Xie \& Yue translated Practical Psychodermatology and published the Chinese version of SPS in the appendix (7). The intensity of itch was evaluated with a $0-10$ numerical rating scale (NRS) (8). Skin lesion severity was evaluated with of Investigator's Static Global Assessment (ISGA) (9) for AD and Lattice System Physician's Global Assessment (LS-PGA) (10) for patients with psoriasis.

The differences in SPS score, age, disease duration and NRS score between $\mathrm{AD}$ and psoriasis patients, and the differences in SPS score between males and females, were compared using a 2-sample $t$-test when normality and homogeneity assumptions were satisfied; otherwise, the Mann-Whitney $U$ test was used. The $\chi^{2}$ test was used to compare sex ratio, the proportion of itching patients, and the proportion of subjects with SPS $\geq 7$ across AD and psoriasis patients. Spearman's correlation was presented to correlate SPS score with age, disease duration, itch intensity and severity of skin lesions. The resulting $p$-values were considered significant if $p<0.05$. Statistical analyses were performed using Statistical Package for Social Science (SPSS 24.0), IBM (Armonk, NY, USA).

Demographic characteristics of survey respondents are shown in Table I. The mean SPS score among patients with AD was $8.69 \pm 4.93$ points (range $0-24$ ), whereas patients with psoriasis scored significantly lower $(4.59 \pm 5.34, p<0.0001)$. The results indicate self-injurious skin picking (SPS $\geq 7)$ in $64.8 \%(n=79)$ of patients with AD and $28.7 \%(n=62)$ of patients with psoriasis $(p<0.0001)$. Most of the patients with self-injurious skin picking (SPS $\geq 7$ ) had intense itching (NRS $\geq 4)$. Mild itching or no itching $(\mathrm{NRS}<4)$ was reported by $11.4 \%(n=9)$ of patients with AD and $6.5 \%(n=4)$ of patients with psoriasis (SPS $\geq 7)$. It was found that these patients' lesions are mainly secondary lesions, such as excoriations and blood scabs.

Patients with AD were more likely to experience itch compared with those with psoriasis $(100 \%$ vs $88 \% ; p<0.001)$. Mean itch severity was higher in subjects with AD compared with subjects with psoriasis (Table I). In patients with $\mathrm{AD}$ and psoriasis, there was no significant difference in SPS scores between different sex $(5.51 \pm 2.88$ vs $6.15 \pm 2.55, p=0.096 ; 4.58 \pm 2.29$ vs $4.63 \pm 2.49$, $p=0.941$; male vs female, respectively), and the proportion of subjects with SPS $\geq 7$ was independent of sex (43.8\% vs $56.2 \%$, $p=0.170 ; 52.9 \%$ vs $47.1 \%, p=0.204$; male vs female, respectively). Severity of itch (NRS) correlated significantly with the SPS

Table I. Demographic and clinical characteristics of survey participants

\begin{tabular}{|c|c|c|c|c|}
\hline Characteristics & Patients with $A D$ & Patients with Ps & $\mathrm{t} / \mathrm{Z}$ & $p$-value \\
\hline \multicolumn{5}{|l|}{ Sex, $n(\%)$} \\
\hline Male & $47(38.50)$ & $153(70.80)$ & & $<0.0001$ \\
\hline Female & $75(61.50)$ & $63(29.20)$ & & \\
\hline Age, years, median [IQR] & $35[26-54]$ & $41[28-57]$ & -1.313 & 0.189 \\
\hline Disease duration, years, median [IQR] & $3[1.50-10]$ & $10[4-20]$ & -5.937 & $<0.001$ \\
\hline $\begin{array}{l}\text { Intensity of itch (numerical rating scale }{ }^{a} \text { ), } \\
\text { mean } \pm S D,(\min -\max )\end{array}$ & $6.91 \pm 2.49,(1-10)$ & $5.00 \pm 2.71,(0-9)$ & 3.143 & $<0.001$ \\
\hline 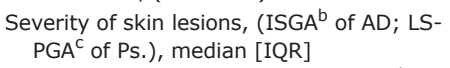 & $3.00[2.00-3.00]$ & $3.00[2.00-5.00]$ & & \\
\hline $\begin{array}{l}\text { Total scale score of Skin Picking Scale }{ }^{d} \\
\text { mean } \pm S D,(\min -\max )\end{array}$ & $8.69 \pm 4.93,(0-23)$ & $4.59 \pm 5.34,(0-21)$ & 7.240 & $<0.0001$ \\
\hline
\end{tabular}

*Significantly different at $p<0.05$.

SD: standard deviation; IQR: interquartile range; AD: atopic dermatitis; Ps: psoriasis; ISGA: Investigator's Static Global Assessment; LS-PGA: Lattice System Physician's Global Assessment.

a Total score range: 0-10; ${ }^{b}$ Total score range: 0-4; ${ }^{\mathrm{C}}$ Total score range: 0-7; Total score range: 0-24. 
Table II Spearman correlation analysis of clinical factors of atopic dermatitis (AD) and psoriasis (Ps) patients with Skin Picking Scale (SPS)

\begin{tabular}{lrrrrrr}
\hline & \multicolumn{2}{c}{ Patients with AD (SPS) } & & \multicolumn{2}{l}{ Patients with PS (SPS) } \\
\cline { 2 - 3 } & r-value & $p$-value & & r-value & $p$-value \\
\hline Age & 0.005 & 0.959 & & 0.312 & 0.061 \\
Disease duration & -0.216 & 0.057 & & 0.246 & 0.067 \\
Severity of skin lesions & 0.290 & 0.051 & & $0.437^{*}$ & $<0.001$ \\
(ISGA of AD; LS-PGA of Ps.) & & & & & & \\
Intensity of itch (NRS) & $0.535^{*}$ & $<0.0001$ & & $0.502^{*}$ & $<0.0001$
\end{tabular}

*Significantly different at $p<0.05$.

ISGA: Investigator's Static Global Assessment; LS-PGA: Lattice System Physician's Global Assessment; NRS: numerical rating scale.

scores both in AD and psoriasis patients (Table II). Age, disease duration and ISGA score did not correlate with the results obtained with SPS (Table II). Nonetheless, LS-PGA score correlated significantly with the SPS score $(r=0.437, p<0.001)$ (Table II).

\section{DISCUSSION}

The current study investigated, for the first time, the selfinjurious skin picking of $\mathrm{AD}$ and psoriasis patients by using SPS. The results show that $64.80 \%$ of patients with $\mathrm{AD}$ and $28.70 \%$ of patients with psoriasis reported selfinjurious skin picking (SPS $\geq 7$ ). This is much higher than the data reported in the community survey; $11.2 \%$ of the total sample reportedly engaged in clinically significant skin picking (SPS $\geq 7$ ) (11). The SPS scores of AD and psoriasis patients were significantly correlated with the degree of pruritus (NRS). Although the sex composition of patients with $\mathrm{AD}$ and psoriasis differs greatly (Table I), there is no sex difference in the SPS scores of the 2 groups of patients. Most of the patients, whose scores of SPS $\geq 7$, had moderate to severe pruritus (NRS $\geq 4$ ), therefore, most patients were considered to be intense pruritus-induced picking. Patients with $\mathrm{AD}$ experience a higher percentage and degree of pruritus than those with psoriasis. This might explain why patients with AD have a higher rate of self-injurious skin picking than patients with psoriasis, and why the proportion of clinically significant skin picking patients is much higher among AD and psoriasis patients than in the community population.

A total of $9(7.38 \%)$ patients with AD and $4(1.85 \%)$ patients with psoriasis, whose SPS $\geq 7$, had mild itching or no itching (NRS $<4$ ), mild primary skin lesions, and obvious secondary rashes, such as scratches, blood scabs, and excoriation. Considering that the uncontrolled picking behaviour and obvious exfoliative skin lesions are not proportional to mild itching, these patients were highly suspicious of comorbid SPD.

In contrast to patients with psoriasis, there is no correlation between skin picking with the degree of the primary skin lesions in patients with AD. This may be explained by the Köbner's phenomenon in psoriasis. Köbner's phenomenon is common in psoriasis, lichen planus and vitiligo and characterized as the appearance of an inflammatory or infectious dermatosis in an area of healthy skin after skin trauma.
In clinical practice, besides skin lesions and itching, more attention should be paid to self-injurious skin picking in $\mathrm{AD}$ and psoriasis patients. In particular, when the skin picking behaviour results in significant tissue damage, distress, and functional impairment, the doctor should provide therapeutic interventions including behavioural cognitive therapy and psychotropic drugs, such as fluoxetine, citalopram, and $\mathrm{N}$-acetylcysteine (12). If necessary, the patient should be referred to a psychiatrist (13).

The prevalence of SPD in the general population ranges from $1.4 \%$ to $5.4 \%$ (14). Acne vulgaris (AV) is the commonest known skin disease combined with SPD (15), but its prevalence is unknown. Other skin diseases, such as AD and psoriasis, may also be combined with SPD, which requires a further large-sample investigation.

This study has some limitations. The sample size was small. Specific skin-picking behaviour and morphological features of skin lesions were not evaluated in detail. In future research, the obsessive-compulsive, stressperceiving, cognition and other psychological conditions of patients with $\mathrm{AD}$ and psoriasis should be evaluated and the correlation with suspicious SPD analysed.

\section{ACKNOWLEDGEMENTS}

The authors are grateful to Hua Zhang, $\mathrm{PhD}$, for statistical evaluation.

The study was approved by the ethics committee of Peking University Third hospital (No. 086-01(2017)).

The authors have no conflicts of interest to declare.

\section{REFERENCES}

1. O'Neill JL, Chan YH, Rapp SR, Yosipovitch G. Differences in itch characteristics between psoriasis and atopic dermatitis patients: results of a web-based questionnaire. Acta Derm Venereol 2011; 91: 537-540.

2. Mochizuki H, Papoiu A, Nattkemper LA, Lin AC, Kraft RA, Coghill RC, et al. Scratching induces overactivity in motorrelated regions and reward system in chronic itch patients. J Invest Dermatol 2015; 135: 2814-2823.

3. Bohne A, Wilhelm S, Keuthen NJ, Baer L, Jenike MA. Skin picking in German students. Prevalence, phenomenology, and associated characteristics. Behav Modif 2002; 26: 320-339.

4. Calikusu C, Yucel B, Polat A, Baykal C. The relation of psychogenic excoriation with psychiatric disorders: a comparative study. Compr Psychiatry 2003; 44: 256-261.

5. Anzengruber F, Ruhwinkel K, Ghosh A, Klaghofer R, Lang UE, Navarini AA. Wide range of age of onset and low referral rates to psychiatry in a large cohort of acne excoriee at a Swiss tertiary hospital. J Dermatolog Treat 2018; 29: 277-280.

6. Keuthen NJ, Wilhelm S, Deckersbach T, Engelhard IM, Forker $A E$, Baer L, et al. The Skin Picking Scale: scale construction and psychometric analyses. J Psychosom Res 2001; 50: 337-341.

7. Appendix Screening questionnaires and scales. In: Bewley A, Taylor RE, Reichenberg JS, Magid M, editors. Practical Psychodermatology. Zhiqiang Xie, Weihua Yue, translated. Beijing: Peking University Medical Press, 2017: p. 313-314.

8. Yosipovitch G, Reaney M, Mastey V, Eckert L, Abbe A, Nelson $L$, et al. Peak Pruritus Numerical Rating Scale: psychometric validation and responder definition for assessing itch in 
moderate-to-severe atopic dermatitis. $\mathrm{Br}$ J Dermatol 2019; 181: 761-769.

9. Paller AS, Tom WL, Lebwohl MG, Blumenthal RL, Boguniewicz M, Call RS, et al. Efficacy and safety of crisaborole ointment, a novel, nonsteroidal phosphodiesterase 4 (PDE4) inhibitor for the topical treatment of atopic dermatitis (AD) in children and adults. J Am Acad Dermatol 2016; 75: 494-503.

10. Langley RG, Ellis CN. Evaluating psoriasis with Psoriasis Area and Severity Index, Psoriasis Global Assessment, and Lattice System Physician's Global Assessment. J Am Acad Dermatol 2004; 51: 563-569.

11. Hayes S L, Storch E A, Berlanga L. Skin picking behaviors: an examination of the prevalence and severity in a community sample[J]. J Anxiety Disorder 2009; 23: 314-319.
12. Jafferany $M$, Patel $A$. skin-picking disorder: a guide to diagnosis and management. Cns Drugs 2019; 33: 337-346.

13. Jafferany M, Mkhoyan R, Arora G, Sadoughifar R, Jorgaqi $E$, Goldust $M$. Treatment of skin picking disorder: interdisciplinary role of dermatologist and psychiatrist. Dermatol Ther 2020; 33: e13837.

14. Torales J, Díaz NR, Barrios I, Navarro R, García O, O’Higgins $M$, et al. Psychodermatology of skin picking (excoriation disorder): a comprehensive review. Dermatol Ther 2020; 33: e13661.

15. Ekore RI, Ekore JO. Excoriation (skin-picking) disorder among adolescents and young adults with acne-induced postinflammatory hyperpigmentation and scars. Int J Dermatol 2021; 60: $1488-1493$. 